\title{
Effects of quetiapine and sertindole on subchronic ketamine-induced deficits in attentional set-shifting in rats
}

\author{
Agnieszka Nikiforuk • Piotr Popik
}

Received: 7 June 2011 / Accepted: 10 August 2011 /Published online: 15 September 2011

(C) The Author(s) 2011. This article is published with open access at Springerlink.com

\begin{abstract}
Rationale Prefrontal cortical dysfunctions, including an impaired ability to shift perceptual attentional set, are core features of schizophrenia. Nevertheless, the effectiveness of second-generation antipsychotic drugs in treating specific prefrontal dysfunctions remains equivocal.

Objectives To model schizophrenia-like cognitive inflexibility in rats, we evaluated the effects of repeated administration of ketamine, the noncompetitive antagonist of the $\mathrm{N}$-methyl-D-aspartate receptor, after a washout period of 14 days in the attentional set-shifting task (ASST). Next, we investigated whether the atypical antipsychotics quetiapine and sertindole would alleviate the ketamine-induced set-shifting impairment.

Methods Ketamine $(30 \mathrm{mg} / \mathrm{kg}$ ) was administered intraperitoneally to rats once daily for 5 or 10 consecutive days to assess its efficacy in producing cognitive impairment. The ASST was performed 14 days following the final drug administration. Quetiapine $(0.63,1.25$ or $2.5 \mathrm{mg} / \mathrm{kg}$ ) or sertindole $(2.5 \mathrm{mg} / \mathrm{kg})$ was administered per os $120 \mathrm{~min}$ before testing.

Results The results of the present study demonstrate that ketamine treatment for 10 but not 5 days significantly and specifically impaired rats' performance in the extradimensional shift (EDs) stage of the ASST. This cognitive inflexibility was reversed by acute administration of sertindole or quetiapine. Quetiapine also promoted setshifting in cognitively unimpaired control animals.

Conclusion The data presented here show that subchronic
\end{abstract}

A. Nikiforuk $(\square) \cdot$ P. Popik

Behavioral Neuroscience and Drug Development,

Institute of Pharmacology, Polish Academy of Sciences,

12 Smetna Street,

31-343 Kraków, Poland

e-mail: nikifor@if-pan.krakow.pl administration of ketamine induces cognitive inflexibility after a washout period. This cognitive deficit likely reflects clinically relevant aspects of cognitive dysfunction encountered in schizophrenic patients. The beneficial effects of quetiapine on set-shifting may have therapeutic implications for the treatment of schizophrenia and other disorders associated with frontal-dependent cognitive impairments.

Keywords Cognitive flexibility - Attentional set-shifting · Ketamine · Quetiapine · Sertindole · Schizophrenia · Animal models $\cdot$ Prefrontal cortex

\section{Introduction}

Deficits of prefrontal cortical function are prominent features of schizophrenia. These neurocognitive dysfunctions include reduced flexibility in modifying behavior in response to the altering relevance of stimuli. This aspect of executive function is commonly assessed in humans using the Wisconsin Card Sorting Test (WCST) (Grant and Berg 1948) and its modified version, the Intradimensional/ Extradimensional Shift (ID/ED) task, developed by Roberts et al. (1988). In fact, a poor WCST and/or ID/ED performance, which is an impairment reminiscent of that observed in patients with frontal lobe damage (Pantelis et al. 1999), is the key cognitive symptom of schizophrenia (Elliott et al. 1998).

Cognitive flexibility may also be assessed in the rodent version of the ID/ED task, i.e., in the attentional set-shifting task (ASST) (Birrell and Brown 2000). In this paradigm, rats must select a bowl containing a food reward based on the ability to discriminate the odors and the media covering the bait. The ASST requires rats to initially learn a rule and form an attentional "set" within the same stimulus 
dimensions. At the extra-dimensional shift (EDs), animals must switch their attention to a new, previously irrelevant stimulus dimension and, for example, discriminate between the odors and no longer between the media covering the bait. The EDs phase, regarded as an index of cognitive flexibility, is impaired by lesions of the medial prefrontal cortex (mPFC) (Birrell and Brown 2000). Hence, the ASST measures specific frontal-dependent cognitive functions in a way homologous to human tests and therefore represents a useful translational approach from animal models to the clinic (Keeler and Robbins 2011).

Noncompetitive antagonists of the $N$-methyl-D-aspartate receptor (NMDAR), such as ketamine and phencyclidine (PCP), produce a behavioral syndrome in healthy humans that closely resembles the symptoms of schizophrenia (Lahti et al. 1999). Therefore, NMDAR-based models are commonly used to mimic a schizophrenia-like state in laboratory animals. Interestingly, ketamine administration to healthy volunteers produced inflexible responding in the WCST, as revealed by an increase in perseverative errors (Krystal et al. 1994). In line with clinical findings, our previous study demonstrated that acute administration of ketamine to rats also impaired their cognitive flexibility during the EDs phase of the ASST (Nikiforuk et al. 2010).

However, although acute administration of NMDAR antagonists evokes a broad range of schizophrenia-like symptoms, experimental data suggest that repeated dosing protocols might represent a more appropriate preclinical approach for modeling neurochemical changes relevant to the pathophysiology of this disorder. Specifically, the repetitive administration of NMDAR antagonists reduced the expression of glutamic acid decarboxylase 67 (GAD67) and the calciumbinding protein, parvalbumin, in cortical aminobutyric acidergic (GABAergic) interneurons (Abdul-Monim et al. 2007). This GABAergic impairment has been regarded as the most consistently demonstrated pathological disturbance in schizophrenia (Lewis and Gonzalez-Burgos 2008). Repeated PCP treatment also evoked a metabolic abnormality, i.e., reduced glucose utilization in the rat PFC, that resembled the hypofrontality commonly observed in schizophrenic patients (Dawson et al. 2010). Additionally, repeated NMDAR antagonist administration leads to dysfunction of cortical dopaminergic transmission in both nonhuman primates and rodents (Jentsch et al. 1997; Jentsch et al. 1998). Because these enduring neurochemical and metabolic alternations in the PFC are thought to underlie cognitive impairments (Dawson et al. 2010; Abdul-Monim et al. 2007; Jentsch et al. 1997), NMDAR antagonist-based preclinical models may represent a useful tool for assessing the pro-cognitive efficacy of antipsychotic drugs.

The first aim of the present study was to investigate the impact of subchronic (5 or 10 consecutive days) administration of the subanesthetic dose $(30 \mathrm{mg} / \mathrm{kg})$ of ketamine on rats' ASST performance assessed after 2 weeks of drug withdrawal. This protocol has been effective for inducing behavioral changes mimicking the positive and negative symptoms of schizophrenia, as revealed by potentiating dizocilpine-induced hyperlocomotion and by the decrease in social behavior, respectively (Becker et al. 2003). Likewise, cognitive deficits were observed in tasks assessing spatial working memory (Enomoto and Floresco 2009) and reversal learning (Floresco et al. 2009). Additionally, second-generation antipsychotic drugs (i.e., clozapine and risperidone) but not typical neuroleptics such as haloperidol ameliorated ketamine-induced impairment of social interaction, suggesting predictive validity of this subchronic treatment paradigm (Becker and Grecksch 2004). In line with persistent behavioral abnormalities, this ketamine dosing schedule also induced dysfunction in GABAergic interneurons (Keilhoff et al. 2004). Therefore, repeated ketamine administration represents a validated model in preclinical schizophrenia research.

Although atypical antipsychotic medications appear to demonstrate a promising effect on cognitive functioning in schizophrenia patients (Harvey and Keefe 2001), several specific dysfunctions including cognitive inflexibility are not normalized by these treatments (Goldberg et al. 2007). Accordingly, atypical antipsychotics including clozapine, olanzapine and risperidone were largely ineffective in normalizing set-shifting deficits in preclinical schizophrenia models (Rodefer et al. 2008; Goetghebeur and Dias 2009; but see also McLean et al. 2008). To date, only sertindole has consistently been shown to reverse cognitive inflexibility (Broberg et al. 2009; Goetghebeur and Dias 2009; Kos et al. 2011; Nikiforuk et al. 2010; Rodefer et al. 2008).

Clinical data indicate that among the newer atypical drugs, quetiapine may possess a distinctive cognitive-enhancing action (Riedel et al. 2010). The pro-cognitive efficacy of quetiapine has been demonstrated in several cognitive domains known to be affected in schizophrenia, including executive functions (Kopala et al. 2006; Purdon et al. 2001; Voruganti et al. 2007). Therefore, the second goal of the present study was to evaluate the effects of quetiapine on the ASST performance of ketamine-treated and control rats; sertindole was used as a positive control.

\section{Materials and methods}

\section{Animals}

Male Sprague-Dawley rats (Charles River, Germany) weighing $250-280 \mathrm{~g}$ on arrival were used in this study. They were initially group-housed $(5 \mathrm{rats} / \mathrm{cage})$ in a temperature- $\left(21 \pm 1^{\circ} \mathrm{C}\right)$ and humidity-controlled (40-50\%) colony room under a 12/12-h light/dark cycle (lights on at 
0600 hours). Rats were allowed to acclimatize for at least 7 days before the start of the experimental procedure. For 1 week prior to testing, rats were individually housed with mild food restriction (15 g of food pellets per day) and ad libitum access to water. Behavioral testing was performed during the light phase of the light/dark cycle. The experiments were conducted in accordance with the NIH Guide for the Care and Use of Laboratory Animals and were approved by the Ethics Committee for Animal Experiments, Institute of Pharmacology.

\section{Attentional set-shifting: apparatus}

Testing was conducted in a modified wire rat housing cage (length $\times$ width $\times$ height: $42 \times 32 \times 22 \mathrm{~cm}$ ) with a white plywood wall dividing half of the length of the cage into two sections (Nikiforuk et al. 2010). During testing, one ceramic digging pot (an internal diameter of $10.5 \mathrm{~cm}$ and a depth of $4 \mathrm{~cm}$ ) was placed in each section. Each pot was defined by a pair of cues along with two stimulus dimensions. To mark each pot with a distinct odor, $5 \mu \mathrm{l}$ of a flavoring essence (Dr. Oetker ${ }^{\circledR}$, Poland) was applied on a piece of blotting paper fixed to the external rim of the pot immediately prior to use. A different pot was used for each combination of digging medium and odor; only one odor was ever applied to a given pot. The bait (one-third of a Honey Nut Cheerio, Nestle ${ }^{\circledR}$ ) was placed at the bottom of the "positive" pot and buried in the digging medium.

\section{Attentional set-shifting: procedure}

The procedure was adapted from Birrell and Brown (Birrell and Brown 2000) and entailed 3 days for each rat.

Day 1, habituation: rats were habituated to the testing area and trained to dig in the pots filled with sawdust to retrieve the food reward. Rats were transported from the housing facility to the testing room where they were presented with one unscented pot (filled with several pieces of Cheerios) in their home cages. After the rats had eaten the Cheerio from the home cage pot, they were placed in the apparatus and given three trials to retrieve the reward from both of the sawdustfilled baited pots. With each exposure, the bait was covered with an increasing amount of sawdust.

Day 2, training: rats were trained on a series of simple discriminations (SD) to a criterion of six consecutive correct trials. For these trials, rats had to learn to associate the food reward with an odor cue (e.g., arrack vs. orange, both pots filled with sawdust) and/or a digging medium (e.g., plastic balls vs. pebbles, no odor). All rats were trained using the same pairs of stimuli. The positive and negative cues for each rat were presented randomly and equally. These training stimuli were not used again in later testing trials.

Day 3, testing: rats performed a series of discriminations in a single test session. The first four trials at the beginning of each discrimination phase were a discovery period (not included in the six criterion trials). In subsequent trials, an incorrect choice was recorded as an error. Digging was defined as any distinct displacement of the digging media with either the paw or the nose; the rat could investigate a digging pot by sniffing or touching without displacing material. Testing was continued at each phase until the rat reached the criterion of six consecutive correct trials, after which testing proceeded to the next phase.

In the simple discrimination (SD) involving only one stimulus dimension, the pots differed along one of two dimensions (i.e., a digging medium). For the compound discrimination (CD), the second (irrelevant) dimension (i.e., an odor) was introduced, but the correct and incorrect exemplars of the relevant dimension remained constant. For the reversal of this discrimination (Rev 1), the exemplars and relevant dimension were unchanged, but the previously correct exemplar was now incorrect and vice versa. The intradimensional (ID) shift was then presented, comprising new exemplars of both the relevant and irrelevant dimensions, with the relevant dimension remaining the same previously. The ID discrimination was then reversed (Rev 2) so that the formerly positive exemplar became the negative one. For the extra-dimensional (ED) shift, a new pair of exemplars was again introduced, but this time a relevant dimension was also changed. Finally, the last phase was the reversal $(\operatorname{Rev} 3)$ of the ED discrimination problem. The exemplars were always presented in pairs and varied so that only one animal within each treatment group received the same combination. The following pairs of exemplars were used: pair 1: odor: lemon vs. almond, medium: cotton wool vs. crumpled tissue; pair 2: odor: spicy vs. vanilla, medium: metallic filler vs. shredded paper; and pair 3: odor: rum vs. cream, medium: clay pellets vs. silk. Our previous study demonstrated that there were no differences in performance of rats shifted from odor to medium and from medium to odor (Nikiforuk et al. 2010). Therefore, in an attempt to simplify the experimental design, the order of discrimination was always the same (i.e., from digging medium to odor). The assignment of each exemplar in a pair as being positive or negative at a given phase and the left-right positioning of the pots in the test apparatus on each trial were randomized.

\section{Drugs}

Ketamine (aqueous solution $(115.34 \mathrm{mg} / \mathrm{ml})$, Biowet, Pulawy, Poland) was diluted in sterile physiological saline. Sertindole (Sigma Aldrich, Poznan, Poland) was dissolved in a minimum amount of 0.1-M hydrochloric acid and diluted with saline, and quetiapine (AstraZeneca) was suspended in $0.5 \%$ methylcellulose.

Ketamine $(30 \mathrm{mg} / \mathrm{kg})$ was administered intraperitoneally to rats once daily for 5 or 10 consecutive days. The ASST 
was performed 14 days following the final drug administration. Animals were left undisturbed during the washout period except for the last 2-3 days when habituation and training were performed. Control animals received vehicle (physiological saline) according to the same experimental schedule. Because no differences were observed between animals receiving vehicle injections for 5 or 10 days, these subgroups were collapsed into one vehicle-treated group for further statistical analysis (Fig. 1).

Sertindole and quetiapine were given per os (PO) $120 \mathrm{~min}$ before testing. Drugs or physiological saline were administered in a volume of $1 \mathrm{ml} / \mathrm{kg}$ of body weight.

The ketamine treatment regime and doses of antipsychotics (i.e., sertindole and quetiapine) were based on previously reported studies (Nikiforuk et al. 2010; Becker et al. 2003; Swerdlow et al. 1996).

\section{Data analysis}

The number of trials required to achieve the criterion of six consecutive correct responses was recorded for each rat and for each discrimination phase. Additionally, the time required by the rats to complete all discriminations was recorded because the compounds could nonspecifically affect performance. The effect of ketamine administration was assessed using two-way ANOVAs with ketamine treatment $(0,5$ and 10 days $)$ as a between-subject factor and discrimination phase (SD, CD, Rev 1, ID, etc.) as a within-subject factor. Data from experiments evaluating the effects of antipsychotic drugs were calculated using three-

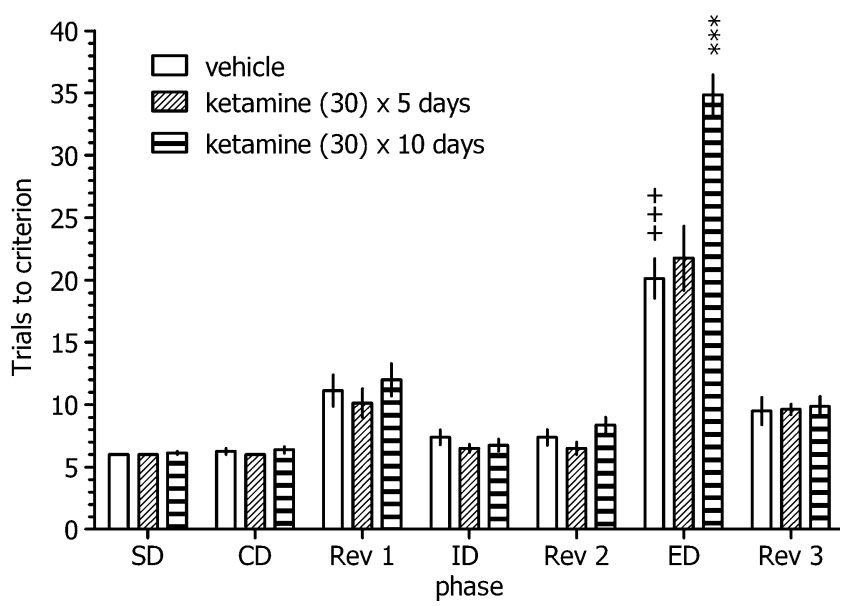

Fig. 1 Enduring debilitating effects of repeated ketamine administration on the performance of the attentional set-shifting task in rats. Ketamine was administered for 5 or 10 consecutive days, and the test was performed 14 days following the last injection. The results represent the mean \pm S.E.M. number of trials required to reach the criterion of six consecutive correct trials for each of the discrimination phases ( $N=8$ rats per group). ${ }^{+++} p<0.001$ vs. vehicle-treated group's IDs performance, $* * * \quad p<0.001$ vs. the vehicle-treated group's EDs performance way ANOVAs with one within-subject factor, i.e., discrimination phase, and two between-subject factors: ketamine treatment ( 0 and 10 days) and a respective antipsychotic drug treatment (sertindole: 0 and $2.5 \mathrm{mg} / \mathrm{kg}$, or quetiapine: $0,0.63,1.25$ and $2.5 \mathrm{mg} / \mathrm{kg}$ ). Post-hoc comparisons were performed using the Newman-Keuls test. The alpha value was set at $p<0.05$. The data fulfilled the criteria for normal distribution. Statistical analyses were performed with the use of Statistica 7.0 for Windows.

\section{Results}

Effect of repeated ketamine administration on rats' performance on the attentional set-shifting task

As illustrated in Fig. 1, there was a statistically significant interaction between ketamine treatment and the discrimination phase: two-way ANOVA interaction factor $F(12,126)=$ $18.26, P<0.0001$. Post-hoc analysis revealed that vehicletreated rats required significantly more trials to reach the criterion on the ED than the ID stage of the task, indicating that they had formed an attentional set towards the relevant dimension before the ED discrimination stage. Furthermore, repeated ketamine administration $(30 \mathrm{mg} / \mathrm{kg}$ ) for 10 (but not 5) consecutive days significantly and specifically impaired rats' performance in the ED stage of the ASST, as indicated by an increased number of trials to criterion during this phase. There was no significant drug effect during any other discrimination stage.

Corresponding to the increased number of trials during the ED phase, 10-day ketamine administration also prolonged the time to complete this discrimination stage (vehicle: $13.5 \pm$ 0.9 min and ketamine $\times 10$ days: $26.3 \pm 0.9$; ANOVA interaction: $F(12,126)=9.71, P<0.0001$; data not shown).

Effect of sertindole on the set-shifting ability of vehicle- and ketamine-treated rats

As illustrated in Fig. 2, there was a statistically significant interaction between ketamine treatment, sertindole treatment and discrimination phase: three-way ANOVA interaction factor $F(6,120)=3.73, P<0.05$. Acute administration of sertindole $(2.5 \mathrm{mg} / \mathrm{kg}, \mathrm{PO})$ reversed the 10 -day ketamineadministration-induced deficit in ED set-shifting performance. In addition, sertindole administration to vehicletreated controls did not produce differences across any of the discrimination phases.

The sertindole-induced facilitation of EDs performance in ketamine-treated rats was associated with reduced time to complete this phase (ketamine-vehicle: $21.3 \pm 1.2 \mathrm{~min}$ and ketamine-sertindole: $13.0 \pm 1.6 \mathrm{~min}$; ANOVA interaction: $F$ $(6,120)=2.43, P<0.05$; data not shown $)$. 


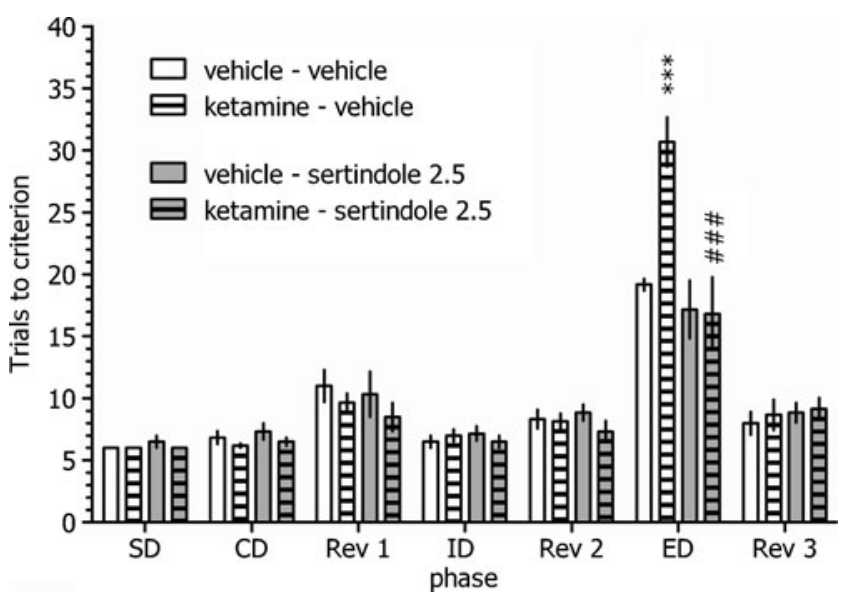

Fig. 2 Effects of sertindole on the deficit in the attentional set-shifting task induced by repeated ketamine administration. Animals received ten daily injections of ketamine. ASST was performed following the 14-day washout period. Sertindole was administered $120 \mathrm{~min}$ before testing; $N=6$ rats per group. $* * * p<0.001$ vs. the vehicle+vehicletreated group's EDs performance, ${ }^{\# \#} p<0.001$ vs. the ketamine +vehicle-treated group's EDs performance

Effect of quetiapine on the set-shifting ability of vehicle- and ketamine-treated rats

As illustrated in Fig. 3, there was a statistically significant interaction between the ketamine treatment, quetiapine treatment and discrimination phase: three-way ANOVA interaction factor $F(18,318)=1.79, P<0.05$. Acute administration of quetiapine $(1.25$ and $2.5 \mathrm{mg} / \mathrm{kg}$, PO) reversed the subchronic ketamine-induced deficit in ED set-shifting performance. Ketamine-treated rats that received quetiapine at a dose of $2.5 \mathrm{mg} / \mathrm{kg}$ also achieved the criterion during the ED stage in fewer trials compared to the vehicle-treated control group. Additionally, quetiapine administration (1.25 and $2.5 \mathrm{mg} / \mathrm{kg}$ ) improved ED set-shifting in vehicle-treated rats. Quetiapine did not affect any other discrimination phase in either vehicle- or ketamine-treated rats.

The improved EDs performance after quetiapine administration to ketamine-treated and control rats was accompanied by the shortened duration of this discrimination phase (vehicle-vehicle: $15.3 \pm 1.1$, vehicle-quetiapine 1.25: $9.4 \pm$ 1.2 and vehicle-quetiapine $2.5: 9.2 \pm 0.7$; ketamine-vehicle: 23.5 \pm 1.5 , ketamine-quetiapine $1.25: 14.2 \pm 1.5$ and ketamine-quetiapine 2.5: $11.45 \pm 1.6$; ANOVA interaction: $F$ $(18,318)=1.87, P<0.05$; data not shown $)$.

\section{Discussion}

The present study reveals that the subchronic administration of ketamine specifically impairs rats' performance on the ED stage of the attentional set-shifting task. Furthermore, this ketamine-induced persistent cognitive inflexibility was reversed by acute quetiapine and sertindole treatment. Quetiapine also facilitated ED set-shifting in cognitively unimpaired control animals.

The present demonstration of ketamine-induced ED setshifting impairment is in line with studies employing repetitive dosing regimens of another NMDAR antagonist, PCP. Indeed, PCP administered twice daily for 7 consecutive days has been consistently demonstrated to impair rats' performance in the ED stage of the ASST following a 10-
Fig. 3 Effects of quetiapine on the deficit in the attentional set-shifting task induced by repeated ketamine administration. Animals received ten daily injections of ketamine, and the test was carried out following the 14-day washout period. Quetiapine was administered 120 min before testing; $N=7-8$ rats per group. $* * * p<0.001$ vs. the vehicle+ vehicle-treated group's EDs performance, ${ }^{\# \#} p<0.001$ vs. the ketamine+vehicle-treated group's EDs performance

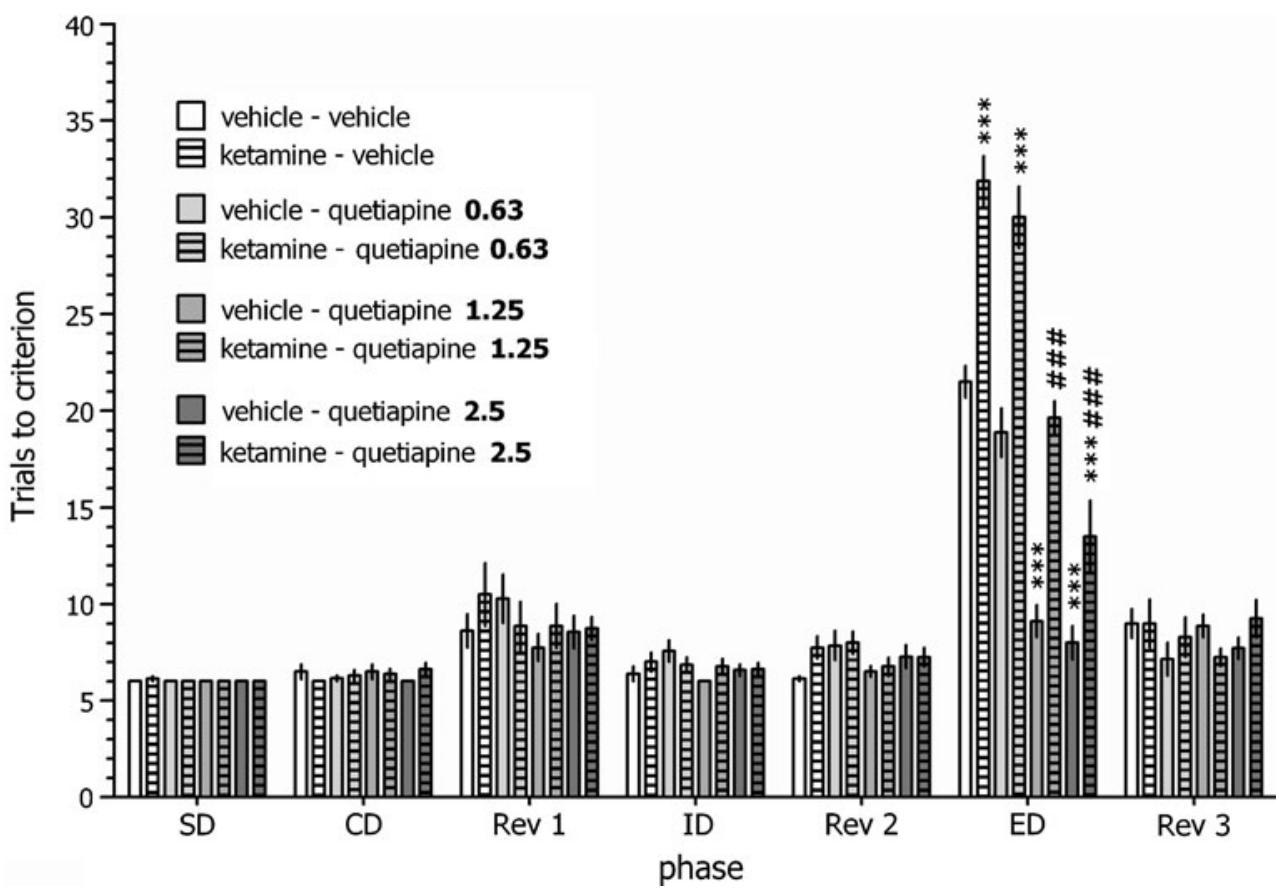


day washout period (Rodefer et al. 2005; Rodefer et al. 2008; Goetghebeur and Dias 2009; McLean et al. 2008). Additionally, Egerton et al. (2008) demonstrated selective impairment of ED set-shifting occurring $72 \mathrm{~h}$ after the last PCP administration following subchronic (5 days) and chronic intermittent treatment regimes. In another study, chronic (14-day) infusion of PCP via osmotic mini-pumps to rats followed by a 7-day drug-free phase also produced selective deficits in the ED stage (Pedersen et al. 2009). The fact that similar results were obtained in neurodevelopmental schizophrenia models, i.e., early post-natal PCP administration (Broberg et al. 2008) or neonatal ventral hippocampal lesions (Marquis et al. 2008), further supports the utility of the ASST in preclinical schizophrenia research.

In contrast to the present findings, using a similar schedule of ketamine administration, Floresco et al. (2009) showed drug-induced deficits in reversal learning during the instrumental and maze-based versions of the set-shifting task. This discrepancy is not surprising in light of the available data. It is known that NMDAR antagonist-induced impairments are common in tasks specifically designed to assess reversal learning ability at the level of changing stimulus-reward contingencies (Abdul-Monim et al. 2007). Conversely, no effect on reversal learning as assessed in the ASST procedure has been demonstrated after either the repeated administration of PCP (Goetghebeur and Dias 2009; Rodefer et al. 2008; Egerton et al. 2005) or acute ketamine treatment (Nikiforuk et al. 2010). As discussed by Floresco et al. (2009), these discrepancies might be due to procedural differences between these paradigms. In contrast to the maze- and operant-based set-shifting protocols that involve extended training, in the ASST, animals usually achieve CD phase in six consecutive trials; this may prevent the formation of a stable stimulus-reward association. Consequently, reversal learning discrimination seems to be relatively easy for animals, as indicated by the high basal level of performance, and is therefore less prone to disruption. However, the results of Floresco et al. (2009) also demonstrated that repeated ketamine administration improved rats' set-shifting performance due to the reduction in perseverative errors. This pattern of responding was similar to that previously noted in rats with inactivation of the nucleus accumbens shell and interpreted as an inability to learn the irrelevance of stimuli during the initial discrimination (Floresco et al. 2006a). Therefore, it is likely that the current protocol is sensitive to different aspects of ketamineevoked set-shifting disruption as compared with the maze and instrumental box protocols.

Alternatively, the differences in ketamine administration protocols may contribute to these discrepancies. Ketamine $(30 \mathrm{mg} / \mathrm{kg}$ ) was administered twice daily for 5 days in the Floresco et al. study and once daily for 10 days in the present experiments. However, Enomoto and Floresco (2009) reported that 5 days of ketamine administration was insufficient to induce PFC-dependent working memory deficits; these occurred only after 10 days of drug administration. Likewise, 5-day ketamine administration did not affect ASST performance in the present study, while the same drug administration protocol has been previously shown to disrupt rats' social behavior and latent inhibition (Becker et al. 2003). Thus, it appears likely that to disrupt mPFC-dependent functions, ketamine should be administered for more than 5 days.

Finally, the potential limitation of the current experiments is the fact that flexibility in shifting attentional set was assessed only in one direction, i.e., from digging medium to odor. However, an initial relevant dimension (odor or medium) did not affect ED set-shifting (Nikiforuk et al. 2010), and it cannot be excluded that ED performance of animals shifted from medium to odor may be more susceptible to the disrupting effects of ketamine.

Sertindole-induced inhibition of the ASST deficit agrees with previous data demonstrating its efficacy in ameliorating ED deficits induced by either subchronic (Broberg et al. 2009; Goetghebeur and Dias 2009; Rodefer et al. 2008) or post-natal (Broberg et al. 2009) PCP administration in rats. Similar beneficial effects of sertindole have been shown in rats (Nikiforuk et al. 2010) and mice (Kos et al. 2011) treated with ketamine acutely, confirming sertindoleinduced improvement of WCST performance in patients with schizophrenia (Gallhofer et al. 2007). Thus, the effectiveness of sertindole in reversing the subchronic ketamine-induced attentional set-shifting deficit may support the predictive validity of the applied model in finding novel compounds that purportedly improve cognitive functioning.

The main finding of the current study is quetiapine-induced facilitation of ED set-shifting; this is the first demonstration of the effectiveness of this drug for modulating prefrontal functions assessed in the ASST. The beneficial effect of quetiapine on cognitive flexibility in ketamine-treated animals may corroborate clinical data, as quetiapine has been demonstrated to improve WCST performance in schizophrenic patients (Kopala et al. 2006; Purdon et al. 2001; Voruganti et al. 2007). However, the lack of drug effects on this specific aspect of executive skills has also been noted (Good et al. 2002; Keefe et al. 2007).

Although quetiapine's effects on set-shifting have not yet been addressed in preclinical studies, existing data suggest its effectiveness in other cognitive domains. For example, Tanibuchi et al. (2009) demonstrated that 2-week administration of quetiapine reversed the deficits caused by repeated PCP administration on a novel object recognition task in mice. Chronic treatment with quetiapine also counteracted the impairment caused by acute PCP administration on rats' 
reference memory in the radial arm maze (He et al. 2006a). Additionally, acute administration of quetiapine restored prepulse inhibition in PCP- and ketamine-treated rats (Swerdlow et al. 1996; Swerdlow et al. 1998). In contrast to its beneficial effects on preattentive information processing, quetiapine treatment did not reverse PCP-induced impairments on attention and impulsivity as assessed in the 5-choice serial reaction time task (Amitai and Markou 2009). Nevertheless, the reported pro-cognitive action of quetiapine was not restricted to schizophrenia-like states. Accordingly, quetiapine treatment ameliorated learning and spatial memory impairments in rats exposed to enhanced single prolonged stress, an animal model of post-traumatic stress disorder (Wang et al. 2010). Quetiapine also alleviated spatial working and reference memory deficits in a transgenic mouse model of Alzheimer's disease (He et al. 2009). Beneficial effects of quetiapine on cognitive functioning were also noted in a cerebral ischemia model (Yan et al. 2007) and in a methamphetamine-induced neurotoxicity model (He et al. 2006b). Correspondingly, the present demonstration of quetiapine-induced facilitation of setshifting in cognitively unimpaired animals may predict its efficacy in disorders other than schizophrenia that are also characterized by disturbances in frontal-dependent cognitive flexibility.

The cognitive benefits of quetiapine in the clinic have been partially ascribed to its relatively low affinity for dopamine D2 receptor together with fast dissociation from these receptors and to the lack of affinity for M1 muscarinic cholinergic receptors (Keefe et al. 2007; Kopala et al. 2006; Riedel et al. 2007; Voruganti et al. 2007). As suggested by Floresco et al. (2006b), D2 receptors may play an important role in regulating set-shifting; the authors demonstrated that the infusion of the D2 receptor antagonist eticlopride into the rat $\mathrm{mPFC}$ disrupted set-shifting ability, as indicated by the increase in perseverative errors (Floresco et al. 2006b). Likewise, systemic administration of the D2 antagonist sulpiride impaired attentional set-shifting in humans (Mehta et al. 2004). Another line of research showed that administration of the muscarinic antagonist scopolamine also impaired rats' performance on the ASST (Chen et al. 2004). Altogether, the unique receptor profile of quetiapine may have an advantage over other antipsychotics.

In contrast to other novel generation antipsychotics (e.g., clozapine) that are characterized by a complex multireceptor profile, quetiapine displays marked affinity only for 5-HT2A, D2, 5-HT1A, $\alpha 1$-adrenergic and $\alpha 2$ adrenergic receptors (Richelson and Souder 2000). Some data indicate that $\alpha 1$-adrenoreceptors might be involved in the mechanism of action of quetiapine. Thus, quetiapine's effectiveness against PCP-induced cognitive deficits in mice (Tanibuchi et al. 2009) and PCP-induced deficits in prepulse inhibition (Bakshi and Geyer 1999) may have resulted from the drug's antagonism at $\alpha 1$-adrenoreceptors. In contrast to this potential therapeutic implication of $\alpha 1$ adrenergic antagonism, the results of Lapiz and Morilak (2006) suggested that $\alpha 1$-receptor stimulation rather than blockade may play a role in facilitating the ASST responding. Thus, it remains questionable if the blockade of $\alpha 1$-adrenergic receptors could improve prefrontal functions specifically related to set-shifting capacity.

Recent data have indicated that the active metabolite of quetiapine, $N$-desalkylquetiapine, may be involved in its actions (Jensen et al. 2008). This compound was characterized as a potent inhibitor of the noradrenaline reuptake transporter (NET) (Jensen et al. 2008) and showed relatively high affinity for the serotonin 7 (5-HT-7) receptor. The pharmacological profile of $N$-desalkylquetiapine, already proposed to explain quetiapine's antidepressant actions, may also account for the drug's pro-cognitive effects. Indeed, NET inhibitors may represent one of the strategies destined to improve prefrontal functions in schizophrenic patients (Apud and Weinberger 2007). In our study, desipramine, a NET inhibitor, facilitated rats' performance in ED set-shifting (Nikiforuk and Popik 2011). Moreover, the selective blockade of 5-HT7 receptors has been shown to be effective for ameliorating cognitive deficits in an NMDAR-based animal model (McLean et al. 2009) and for improving cognitive flexibility in rats (our unpublished data). Therefore, one can assume that the activities of $N$-desalkylquetiapine at the NET and/or 5-HT7 receptors may contribute to the pro-cognitive action of quetiapine in the ASST.

It has been proposed that the ability of atypical antipsychotic drugs to increase catecholamine levels in the prefrontal cortex might underlie their potential pro-cognitive action. Likewise, quetiapine has been demonstrated to increase dopamine and noradrenaline levels in the rat $\mathrm{mPFC}$ (Ichikawa et al. 2002; Pira et al. 2004; Yamamura et al. 2009). However, the lack of an effect of quetiapine on PFC dopamine release was also noted (Denys et al. 2004). Because medications enhancing frontal dopaminergic and noradrenergic neurotransmission were shown to facilitate ED set-shifting (Nikiforuk et al. 2010; Nikiforuk and Popik 2011; Tunbridge et al. 2004), the quetiapine-evoked increase in catecholamine levels could account for its beneficial action in the ASST. However, it should be noted that novel generation antipsychotic drugs (i.e., risperidone and olanzapine) increased the prefrontal dopamine level but had no effect on the setshifting task (Rodefer et al. 2008), suggesting that the mere enhancement of dopamine release is insufficient to produce facilitation of set-shifting. Furthermore, similarly to the actions of sertindole but not risperidone (Mork et al. 2009), quetiapine enhances the cortical glutamate level (Yamamura et al. 2009). The role of glutamatergic transmission in modulating cognitive flexibility has previously been suggested (Nikiforuk 
et al. 2011). Finally, elevation of the acetylcholine level in the $\mathrm{mPFC}$ was also noted after quetiapine administration (Ichikawa et al. 2002). Nevertheless, it remains equivocal whether cholinergic transmission plays a critical role in the modulation of attentional set-shifting because acetylcholine depletion in the rat $\mathrm{mPFC}$ did not affect performance on that task (McGaughy et al. 2008).

In summary, the present demonstration of the subchronic ketamine-induced attentional set-shifting deficit reflects clinically relevant aspects of cognitive dysfunction encountered in schizophrenic patients. Furthermore, the effectiveness of sertindole in reversing ketamine-induced cognitive inflexibility may support the predictive validity of the applied model. Finally, the beneficial effects of quetiapine on ASST performance may have therapeutic implications for the treatment of schizophrenia and other disorders associated with frontal-dependent cognitive impairments.

Acknowledgments This study was supported by the Statutory Funds of the Institute of Pharmacology, the Polish Academy of Sciences and project "Prokog," UDA-POIG.01.03.01-12-063/09-00, co-financed by the European Union from the European Fund of Regional Development.

Open Access This article is distributed under the terms of the Creative Commons Attribution Noncommercial License which permits any noncommercial use, distribution, and reproduction in any medium, provided the original author(s) and source are credited.

\section{References}

Abdul-Monim Z, Neill JC, Reynolds GP (2007) Sub-chronic psychotomimetic phencyclidine induces deficits in reversal learning and alterations in parvalbumin-immunoreactive expression in the rat. J Psychopharmacol 21:198-205

Amitai N, Markou A (2009) Increased impulsivity and disrupted attention induced by repeated phencyclidine are not attenuated by chronic quetiapine treatment. Pharmacol Biochem Behav 93:248-257

Apud JA, Weinberger DR (2007) Treatment of cognitive deficits associated with schizophrenia: potential role of catechol-Omethyltransferase inhibitors. CNS Drugs 21:535-557

Bakshi VP, Geyer MA (1999) Alpha-1-adrenergic receptors mediate sensorimotor gating deficits produced by intracerebral dizocilpine administration in rats. Neuroscience 92:113-121

Becker A, Grecksch G (2004) Ketamine-induced changes in rat behaviour: a possible animal model of schizophrenia. Test of predictive validity. Prog Neuropsychopharmacol Biol Psychiatry 28:1267-1277

Becker A, Peters B, Schroeder H, Mann T, Huether G, Grecksch G (2003) Ketamine-induced changes in rat behaviour: a possible animal model of schizophrenia. Prog Neuropsychopharmacol Biol Psychiatry 27:687-700

Birrell JM, Brown VJ (2000) Medial frontal cortex mediates perceptual attentional set shifting in the rat. J Neurosci 20:4320-4324

Broberg BV, Dias R, Glenthoj BY, Olsen CK (2008) Evaluation of a neurodevelopmental model of schizophrenia - early postnatal PCP treatment in attentional set-shifting. Behavioural Brain Research 190:160-163

Broberg BV, Glenthoj BY, Dias R, Larsen DB, Olsen CK (2009) Reversal of cognitive deficits by an ampakine (CX516) and sertindole in two animal models of schizophrenia - sub-chronic and early postnatal PCP treatment in attentional set-shifting. Psychopharmacology (Berl) 206:631-640

Chen KC, Baxter MG, Rodefer JS (2004) Central blockade of muscarinic cholinergic receptors disrupts affective and attentional set-shifting. Eur J Neurosci 20:1081-1088

Dawson N, Thompson RJ, McVie A, Thomson DM, Morris BJ, Pratt JA (2010) Modafinil reverses phencyclidine-induced deficits in cognitive flexibility, cerebral metabolism, and functional brain connectivity. Schizophr Bull. doi:10.1093/schbul/sbq090

Denys D, Klompmakers AA, Westenberg HG (2004) Synergistic dopamine increase in the rat prefrontal cortex with the combination of quetiapine and fluvoxamine. Psychopharmacology (Berl) 176:195-203

Egerton A, Reid L, McGregor S, Cochran SM, Morris BJ, Pratt JA (2008) Subchronic and chronic PCP treatment produces temporally distinct deficits in attentional set shifting and prepulse inhibition in rats. Psychopharmacology (Berl) 198:37-49

Egerton A, Reid L, McKerchar CE, Morris BJ, Pratt JA (2005) Impairment in perceptual attentional set-shifting following PCP administration: a rodent model of set-shifting deficits in schizophrenia. Psychopharmacology (Berl) 179:77-84

Elliott R, McKenna PJ, Robbins TW, Sahakian BJ (1998) Specific neuropsychological deficits in schizophrenic patients with preserved intellectual function. Cognitive Neuropsychiatry 3:45-70

Enomoto T, Floresco SB (2009) Disruptions in spatial working memory, but not short-term memory, induced by repeated ketamine exposure. Prog Neuropsychopharmacol Biol Psychiatry 15:668-675

Floresco SB, Ghods-Sharifi S, Vexelman C, Magyar O (2006a) Dissociable roles for the nucleus accumbens core and shell in regulating set shifting. J Neurosci 26:2449-2457

Floresco SB, Magyar O, Ghods-Sharifi S, Vexelman C, Tse MT (2006b) Multiple dopamine receptor subtypes in the medial prefrontal cortex of the rat regulate set-shifting. Neuropsychopharmacology 31:297-309

Floresco SB, Zhang Y, Enomoto T (2009) Neural circuits subserving behavioral flexibility and their relevance to schizophrenia. Behav Brain Res 204:396-409

Gallhofer B, Jaanson P, Mittoux A, Tanghoj P, Lis S, Krieger S (2007) Course of recovery of cognitive impairment in patients with schizophrenia: a randomised double-blind study comparing sertindole and haloperidol. Pharmacopsychiatry 40:275-286

Goetghebeur P, Dias R (2009) Comparison of haloperidol, risperidone, sertindole, and modafinil to reverse an attentional set-shifting impairment following subchronic PCP administration in the rat-a back translational study. Psychopharmacology (Berl) 202:287293

Goldberg TE, Goldman RS, Burdick KE, Malhotra AK, Lencz T, Patel RC, Woerner MG, Schooler NR, Kane JM, Robinson DG (2007) Cognitive improvement after treatment with second-generation antipsychotic medications in first-episode schizophrenia: is it a practice effect? Arch Gen Psychiatry 64:1115-1122

Good KP, Kiss I, Buiteman C, Woodley H, Rui Q, Whitehorn D, Kopala L (2002) Improvement in cognitive functioning in patients with first-episode psychosis during treatment with quetiapine: an interim analysis. Br J Psychiatry Suppl 43:s45-s49

Grant DA, Berg EA (1948) A behavioral analysis of degree of reinforcement and ease of shifting to new responses in a Weigltype card-sorting problem. J Exp Psychol 38:404-411

Harvey PD, Keefe RS (2001) Studies of cognitive change in patients with schizophrenia following novel antipsychotic treatment. Am J Psychiatry 158:176-184

He J, Luo H, Yan B, Yu Y, Wang H, Wei Z, Zhang Y, Xu H, Tempier A, Li X, Li XM (2009) Beneficial effects of quetiapine in a transgenic mouse model of Alzheimer's disease. Neurobiol Aging 30:1205-1216 
He J, Xu H, Yang Y, Rajakumar D, Li X, Li XM (2006a) The effects of chronic administration of quetiapine on the phencyclidineinduced reference memory impairment and decrease of Bcl-XL/ Bax ratio in the posterior cingulate cortex in rats. Behav Brain Res 168:236-242

He J, Yang Y, Yu Y, Li X, Li XM (2006b) The effects of chronic administration of quetiapine on the methamphetamine-induced recognition memory impairment and dopaminergic terminal deficit in rats. Behav Brain Res 172:39-45

Ichikawa J, Li Z, Dai J, Meltzer HY (2002) Atypical antipsychotic drugs, quetiapine, iloperidone, and melperone, preferentially increase dopamine and acetylcholine release in rat medial prefrontal cortex: role of 5-HT1A receptor agonism. Brain Res 956:349-357

Jensen NH, Rodriguiz RM, Caron MG, Wetsel WC, Rothman RB, Roth BL (2008) $N$-desalkylquetiapine, a potent norepinephrine reuptake inhibitor and partial 5-HT1A agonist, as a putative mediator of quetiapine's antidepressant activity. Neuropsychopharmacology 33:2303-2312

Jentsch JD, Dazzi L, Chhatwal JP, Verrico CD, Roth RH (1998) Reduced prefrontal cortical dopamine, but not acetylcholine, release in vivo after repeated, intermittent phencyclidine administration to rats. Neurosci Letter 258:175-178

Jentsch JD, Redmond DE, Elsworth JD, Taylor JR, Youngren KD, Roth RH (1997) Enduring cognitive deficits and cortical dopamine dysfunction in monkeys after long-term administration of phencyclidine. Science 277:953-955

Keefe RS, Sweeney JA, Gu H, Hamer RM, Perkins DO, McEvoy JP, Lieberman JA (2007) Effects of olanzapine, quetiapine, and risperidone on neurocognitive function in early psychosis: a randomized, double-blind 52-week comparison. Am J Psychiatry 164:1061-1071

Keeler JF, Robbins TW (2011) Translating cognition from animals to humans. Biochem Pharmacol 81:1356-1366

Keilhoff G, Becker A, Grecksch G, Wolf G, Bernstein HG (2004) Repeated application of ketamine to rats induces changes in the hippocampal expression of parvalbumin, neuronal nitric oxide synthase and cFOS similar to those found in human schizophrenia. Neuroscience 126:591-598

Kopala LC, Good KP, Milliken H, Buiteman C, Woodley H, Rui Q, Whitehorn D, Love L, Balshaw R, Kiss I, Honer WG (2006) Treatment of a first episode of psychotic illness with quetiapine: an analysis of 2 year outcomes. Schizophr Res 81:29-39

Kos T, Nikiforuk A, Rafa D, Popik P (2011) The effects of NMDA receptor antagonists on attentional set-shifting task performance in mice. Psychopharmacology (Berl) 214:911-921

Krystal JH, Karper LP, Seibyl JP, Freeman GK, Delaney R, Bremner JD, Heninger GR, Bowers MB, Charney DS (1994) Subanesthetic effects of the noncompetitive NMDA antagonist, ketamine, in humans. Psychotomimetic, perceptual, cognitive, and neuroendocrine responses. Archives of General Psychiatry 51:199-214

Lahti AC, Holcomb HH, Gao XM, Tamminga CA (1999) NMDAsensitive glutamate antagonism: a human model for psychosis. Neuropsychopharmacology 21:S158-S169

Lapiz MD, Morilak DA (2006) Noradrenergic modulation of cognitive function in rat medial prefrontal cortex as measured by attentional set shifting capability. Neuroscience 137:1039-1049

Lewis DA, Gonzalez-Burgos G (2008) Neuroplasticity of neocortical circuits in schizophrenia. Neuropsychopharmacology 33:141-165

Marquis JP, Goulet S, Dore FY (2008) Neonatal ventral hippocampus lesions disrupt extra-dimensional shift and alter dendritic spine density in the medial prefrontal cortex of juvenile rats. Neurobiol Learn Mem 90:339-346

McGaughy J, Ross RS, Eichenbaum H (2008) Noradrenergic, but not cholinergic, deafferentation of prefrontal cortex impairs attentional set-shifting. Neuroscience 153:63-71
McLean SL, Beck JP, Woolley ML, Neill JC (2008) A preliminary investigation into the effects of antipsychotics on sub-chronic phencyclidine-induced deficits in attentional set-shifting in female rats. Behav Brain Res 189:152-158

McLean SL, Woolley ML, Thomas D, Neill JC (2009) Role of 5-HT receptor mechanisms in sub-chronic PCP-induced reversal learning deficits in the rat. Psychopharmacology (Berl) 206:403-414

Mehta MA, Manes FF, Magnolfi G, Sahakian BJ, Robbins TW (2004) Impaired set-shifting and dissociable effects on tests of spatial working memory following the dopamine D2 receptor antagonist sulpiride in human volunteers. Psychopharmacology (Berl) 176:331-342

Mork A, Witten LM, Arnt J (2009) Effect of sertindole on extracellular dopamine, acetylcholine, and glutamate in the medial prefrontal cortex of conscious rats: a comparison with risperidone and exploration of mechanisms involved. Psychopharmacology (Berl) 206:39-49

Nikiforuk A, Golembiowska K, Popik P (2010) Mazindol attenuates ketamine-induced cognitive deficit in the attentional set shifting task in rats. Eur Neuropsychopharmacol 20:37-48

Nikiforuk A, Kos T, Rafa D, Behl B, Bespalov A, Popik P (2011) Blockade of glycine transporter 1 by SSR-504734 promotes cognitive flexibility in glycineB/NMDA receptor-dependent manner. Neuropharmacology 61:262-267

Nikiforuk A, Popik P (2011) Long-lasting cognitive deficit induced by stress is alleviated by acute administration of antidepressants. Psychoneuroendocrinology 36:28-39

Pantelis C, Barber FZ, Barnes TR, Nelson HE, Owen AM, Robbins TW (1999) Comparison of set-shifting ability in patients with chronic schizophrenia and frontal lobe damage. Schizophr Res $37: 251-270$

Pedersen CS, Goetghebeur P, Dias R (2009) Chronic infusion of PCP via osmotic mini-pumps: a new rodent model of cognitive deficit in schizophrenia characterized by impaired attentional setshifting (ID/ED) performance. J Neurosci Methods 185:66-69

Pira L, Mongeau R, Pani L (2004) The atypical antipsychotic quetiapine increases both noradrenaline and dopamine release in the rat prefrontal cortex. Eur J Pharmacol 504:61-64

Purdon SE, Malla A, Labelle A, Lit W (2001) Neuropsychological change in patients with schizophrenia after treatment with quetiapine or haloperidol. J Psychiatry Neurosci 26:137-149

Richelson E, Souder T (2000) Binding of antipsychotic drugs to human brain receptors focus on newer generation compounds. Life Sci 68:29-39

Riedel M, Muller N, Spellmann I, Engel RR, Musil R, Valdevit R, Dehning S, Douhet A, Cerovecki A, Strassnig M, Moller HJ (2007) Efficacy of olanzapine versus quetiapine on cognitive dysfunctions in patients with an acute episode of schizophrenia. Eur Arch Psychiatry Clin Neurosci 257:402-412

Riedel M, Schennach-Wolff R, Musil R, Dehning S, Cerovecki A, Opgen-Rhein M, Matz J, Seemuller F, Obermeier M, Engel RR, Muller N, Moller HJ, Spellmann I (2010) Neurocognition and its influencing factors in the treatment of schizophrenia-effects of aripiprazole, olanzapine, quetiapine and risperidone. Hum Psychopharmacol 25:116-125

Roberts AC, Robbins TW, Everitt BJ (1988) The effects of intradimensional and extradimensional shifts on visual discrimination learning in humans and non-human primates. Q J Exp Psychol B 40:321-341

Rodefer JS, Murphy ER, Baxter MG (2005) PDE10A inhibition reverses subchronic PCP-induced deficits in attentional setshifting in rats. Eur J Neurosci 21:1070-1076

Rodefer JS, Nguyen TN, Karlsson JJ, Arnt J (2008) Reversal of subchronic PCP-induced deficits in attentional set shifting in rats by sertindole and a 5-HT(6) receptor antagonist: comparison among antipsychotics. Neuropsychopharmacology 33:26572666 
Swerdlow NR, Bakshi V, Geyer MA (1996) Seroquel restores sensorimotor gating in phencyclidine-treated rats. J Pharmacol Exp Ther 279:1290-9

Swerdlow NR, Bakshi V, Waikar M, Taaid N, Geyer MA (1998) Seroquel, clozapine and chlorpromazine restore sensorimotor gating in ketamine-treated rats. Psychopharmacology (Berl) 140:75-80

Tanibuchi Y, Fujita Y, Kohno M, Ishima T, Takatsu Y, Iyo M, Hashimoto K (2009) Effects of quetiapine on phencyclidineinduced cognitive deficits in mice: a possible role of alpha1adrenoceptors. Eur Neuropsychopharmacol 19:861-867

Tunbridge EM, Bannerman DM, Sharp T, Harrison PJ (2004) Catechol-o-methyltransferase inhibition improves set-shifting performance and elevates stimulated dopamine release in the rat prefrontal cortex. J Neurosci 24:5331-5335

Voruganti LP, Awad AG, Parker G, Forrest C, Usmani Y, Fernando ML, Senthilal S (2007) Cognition, functioning and quality of life in schizophrenia treatment: results of a one-year randomized controlled trial of olanzapine and quetiapine. Schizophr Res 96:146-155

Wang HN, Peng Y, Tan QR, Chen YC, Zhang RG, Qiao YT, Wang HH, Liu L, Kuang F, Wang BR, Zhang ZJ (2010) Quetiapine ameliorates anxiety-like behavior and cognitive impairments in stressed rats: implications for the treatment of posttraumatic stress disorder. Physiol Res 59:263-271

Yamamura S, Ohoyama K, Hamaguchi T, Kashimoto K, Nakagawa M, Kanehara S, Suzuki D, Matsumoto T, Motomura E, Shiroyama T, Okada M (2009) Effects of quetiapine on monoamine, GABA, and glutamate release in rat prefrontal cortex. Psychopharmacology (Berl) 206:243-258

Yan B, Bi X, He J, Zhang Y, Thakur S, Xu H, Gendron A, Kong J, Li XM (2007) Quetiapine attenuates spatial memory impairment and hippocampal neurodegeneration induced by bilateral common carotid artery occlusion in mice. Life Sci 81:353-361 